\title{
Leucovorin and fluorouracil plus oxaliplatin or leucovorin and fluorouracil plus irinotecan as individualized first-line therapy based on a drug sensitivity test
}

\author{
TAKUMI OCHIAI ${ }^{1}$, KAZUHIKO NISHIMURA $^{1}$, TOMOO WATANABE ${ }^{1}$, MASAYUKI KITAJIMA $^{1}$, \\ TADASUKE HASHIGUCHI ${ }^{1}$, AKINORI NAKATANI ${ }^{1}$, TAKASHI MARUSASA ${ }^{1}$, \\ AKIRA MURAKI $^{1}$, ISAO NAGAOKA ${ }^{2}$ and SHUNJI FUTAGAWA ${ }^{1}$ \\ ${ }^{1}$ Department of Surgery, Tobu Chiiki Hospital, Tokyo Metropolitan Health and Medical Treatment Corporation; \\ ${ }^{2}$ Department of Host Defense and Biochemical Research, Juntendo University School of Medicine, Tokyo, Japan
}

Received October 16, 2009; Accepted December 8, 2009

DOI: 10.3892/etm_00000050

\begin{abstract}
The purpose of this study was to determine the effect of the addition of oxaliplatin (l-OHP) or irinotecan (SN-38) to 5-fluorouracil (5-FU) using the collagen gel droplet embedded culture-drug sensitivity test (CD-DST) to establish whether leucovorin plus 5-FU should be administered in combination with $l$-OHP (FOLFOX) or SN-38 (FOLFIRI) in individualized first-line chemotherapy for the treatment of advanced colorectal cancer (CRC). Specimens of primary tumors were obtained from 24 CRC patients who had received no preoperative chemotherapy. CD-DST was performed, and the inhibition rate (IR) was obtained under multiple incubation conditions. The effects of addition of $l$-OHP or SN-38 were evaluated for the same area under the concentration curve (AUC) of 5-FU based on linear regression analysis. Approximate expression and correlation coefficients (5-FU vs. 5 -FU $+l$-OHP, 5 -FU vs. $5-\mathrm{FU}+\mathrm{SN}-38$; AUC of $5-\mathrm{FU}=72$ and 5 -FU vs. $5-\mathrm{FU}+l$-OHP, $5-\mathrm{FU}$ vs. $5-\mathrm{FU}+\mathrm{SN}-38$; AUC of 5 -FU=144) were $\mathrm{y}=0.94 \mathrm{x}+8.53 \quad\left(\mathrm{R}^{2}=0.95, \mathrm{p}<0.0004\right)$, $\mathrm{y}=0.77 \mathrm{x}+26.18 \quad\left(\mathrm{R}^{2}=0.76, \quad \mathrm{p}<0.0004\right) \quad$ and $\mathrm{y}=0.91 \mathrm{x}+10.90$ $\left(\mathrm{R}^{2}=0.94, \mathrm{p}<0.0004\right), \mathrm{y}=0.52 \mathrm{x}+44.61 \quad\left(\mathrm{R}^{2}=0.60, \mathrm{p}<0.0004\right)$, respectively. Approximate expression of 5-FU vs. 5-FU + $l$-OHP almost fit the regression line $\left(\mathrm{y}=\mathrm{x}+\mathrm{b}_{1}\right)$. This suggests that addition of $l$-OHP yields a constant additive effect, independent of the IR of 5-FU. However, approximate expression of 5-FU vs. 5-FU $+\mathrm{SN}-38$ fit the regression line $\left(y=a x+b_{2}, a<1, b_{2} \geq b_{1}\right)$. This suggests that addition of SN-38
\end{abstract}

Correspondence to: Dr Takumi Ochiai, Department of Surgery, Tobu Chiiki Hospital Tokyo Metropolitan Health and Medical Treatment Corporation, 5-14-1 Kameari, Katsushika-ku, Tokyo 125-8512, Japan

E-mail: takumi-o@ma.kitanet.ne.jp

Key words: colorectal cancer, individualized chemotherapy, collagen gel droplet embedded drug sensitivity test, FOLFOX, FOLFIRI yields a greater additive effect due to the lower IR of 5-FU. These results indicate that FOLFIRI should be selected as the first-line chemotherapy for the treatment of poor responders to $5-\mathrm{FU}$.

\section{Introduction}

Over the last decade, leucovorin (FOL) and 5-fluorouracil (5-FU) plus oxaliplatin (l-OHP) (FOLFOX) or leucovorin and 5-FU plus irinotecan (SN-38) (FOLFIRI) have been widely used as first-line chemotherapy in the treatment of advanced colorectal cancer (CRC). Moreover, molecularly targeted drugs such as bevacizumab and cetuximab have improved overall and progression-free patient survival. In general, it is not important whether FOLFOX or FOLFIRI is administered first. However, in order to achieve improvements in terms of survival, it is crucial to achieve full administration of the targeted dosages of all 3 drugs; leucovorin and 5-FU plus $l$-OHP or SN-38.

Where possible, the most effective regimen should be chosen as the first line of treatment. In an earlier study, we reported that the area under the concentration curve (AUC) and growth inhibition rate (IR) were combined to give the AUC-IR curve, which was approximated to the logarithmic curve for 5-FU. Moreover, our results from the collagen gel droplet embedded culture-drug sensitivity test (CD-DST) indicated that the anti-tumor effect of 5-FU depended on the AUC in CRC (1). In another study, we reported that individual $50 \%$ inhibitory AUCs could be calculated from individual AUC-IR regression curves (2).

The aim of this study was to evaluate the effect of the addition of $l$-OHP or SN-38 to 5-FU using the CD-DST to establish whether FOLFOX or FOLFIRI should be chosen in individualized chemotherapy for the first-line treatment of advanced CRC.

\section{Materials and methods}

Patients. Specimens of primary tumors were obtained from 24 CRC patients who had received no preoperative chemotherapy 
between March 2008 and April 2009. Informed consent for measuring drug sensitivity was obtained from all patients.

Methods. Tumor tissue was excised from primary surgical specimens and subjected to the CD-DST. The CD-DST was used to evaluate the sensitivity of tumors to 5-FU and was performed as described by Kobayashi et al $(3,4)$. Each specimen was washed 5 times with $50 \mathrm{ml}$ saline, followed by further washing 5 times with $50 \mathrm{ml}$ antibiotic fluid containing $1.0 \mathrm{mg} / \mathrm{ml}$ piperacillin and $0.5 \mathrm{mg} / \mathrm{ml} \mathrm{kanamycin.} \mathrm{The}$ transport bottle contained $1.0 \mathrm{mg} / \mathrm{ml}$ piperacillin, $0.5 \mathrm{mg} / \mathrm{ml}$ kanamycin and $2.5 \mu \mathrm{g} / \mathrm{ml}$ amphotericin B.

Tissue $(1 \mathrm{~g})$ was treated with a dispersion enzyme cocktail containing $1.0 \%$ collagenase for $2 \mathrm{~h}$. Dispersed cell suspensions were inoculated into pre-culture media on collagen-coated flasks overnight, after which viable tumor cells were recovered by $0.05 \%$ collagenase treatment. Recovered cells were embedded in 30- $\mu$ l collagen gel droplets. Embedded cells were cultivated in culture media containing 5-FU at $10.0 \mu \mathrm{g}$ for $24 \mathrm{~h}, 3.0 \mu \mathrm{g}$ for $24 \mathrm{~h}, 1.0 \mu \mathrm{g}$ for $120 \mathrm{~h}$ and $0.2 \mu \mathrm{g}$ for $24 \mathrm{~h}$; $5-\mathrm{FU}+l-\mathrm{OHP}$ at $3.0+1.5 \mu \mathrm{g}$ and $6.0+3.0 \mu \mathrm{g}$, respectively, for $24 \mathrm{~h}$; and 5-FU $+\mathrm{SN}-38$ at $3.0+0.1 \mu \mathrm{g}$ and $6.0+0.2 \mu \mathrm{g}$, respectively, for $24 \mathrm{~h}$.

After removal of 5-FU-containing media, cells were further cultured for 7 days in serum-free culture media to prevent growth of fibroblasts. Viable cells were stained with neutral red solution and counted by the imaging colorimetric quantification method. Surviving cell number ratio between the drug-treated group and the control group which received no drug treatment was calculated. A growth rate in excess of 0.8 was considered indicative of successful culture.

After converting drug concentration and contact time to an AUC, an AUC-IR curve was plotted against the growth IR. The effect of the individual growth IR on the AUC $(144 \mu \mathrm{g} * \mathrm{~h} /$ $\mathrm{ml}$ ) of 5-FU was calculated from the AUC-IR regression curve. The effect of the addition of $l$-OHP or SN-38 on the same AUC was evaluated.

Statistical analysis. Comparisons of the growth IR value between 5 -FU and 5 -FU $+l$-OHP or $\mathrm{SN}-38$ were compiled using the paired t-test. Correlations between the growth IRs of 5 -FU and $5-\mathrm{FU}+l$-OHP or SN-38 on the same AUC of 5 -FU were analyzed by linear regression analysis. Statistical tests were carried out using the SPSS package (version II for Windows). P-values $<0.05$ were regarded as statistically significant.

\section{Results}

The AUC-IR curve of a representative patient is shown in Fig. 1. Approximate expression and correlation coefficients were $\mathrm{y}=14.012 \mathrm{Ln}(\mathrm{x})+2.4063\left(\mathrm{R}^{2}=0.9719\right)$ for $\mathrm{AUC}$ and $72.0 \%$ for the individual growth IR value on the AUC $(144 \mu \mathrm{g} * \mathrm{~h} / \mathrm{ml})$ of 5-FU calculated from the regression curve of this patient.

The growth IR value on the AUC $(144 \mu \mathrm{g} * \mathrm{~h} / \mathrm{ml})$ of $5-\mathrm{FU}$ was calculated to give the AUC-IR curve for each patient (Table I).

Use of $l$-OHP or SN-38 in combination with 5-FU yielded a significant increase in the growth IR value on the AUC of 5-FU (Table I).

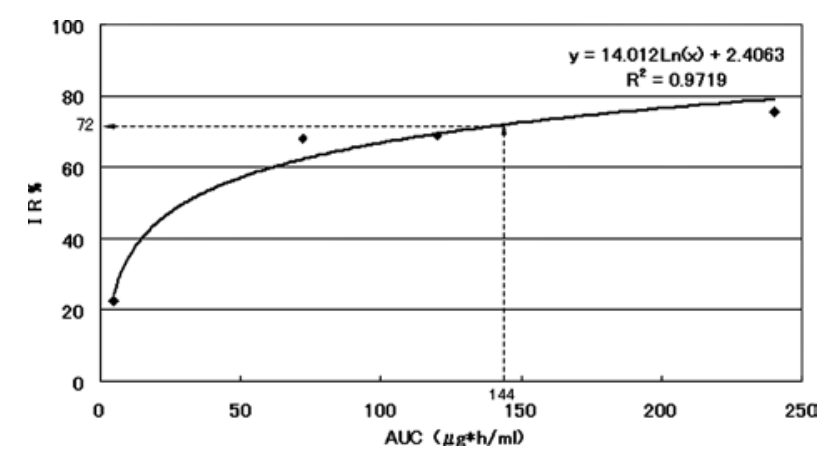

Figure 1. Growth inhibition according to representative 5-fluorouracil (5-FU) area under the curve (AUC). Seventy-two percent for the individual growth inhibition rate (IR) value on the AUC $(144 \mu \mathrm{g} * \mathrm{~h} / \mathrm{ml})$ of 5 -FU was calculated from the regression curve of this patient.

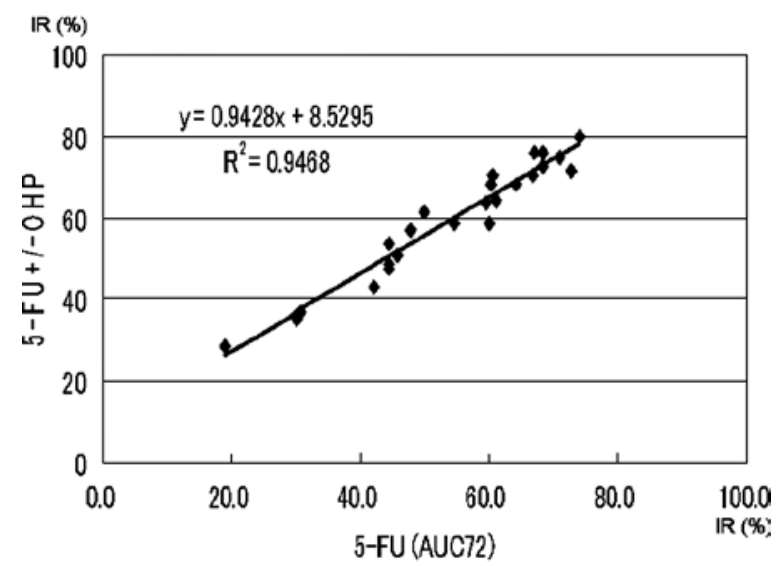

Figure 2. Effect of the addition of oxaliplatin ( $l$-OHP) to 5-fluorouracil (5-FU) on linear regression analysis. The growth inhibition rate (IR) of embedded cancer cells cultivated in culture media containing 5-FU at $3.0 \mu \mathrm{g}$ for $24 \mathrm{~h}$ vs. $5-\mathrm{FU}+l$-OHP at $3.0+1.5 \mu \mathrm{g}$ for $24 \mathrm{~h}$.

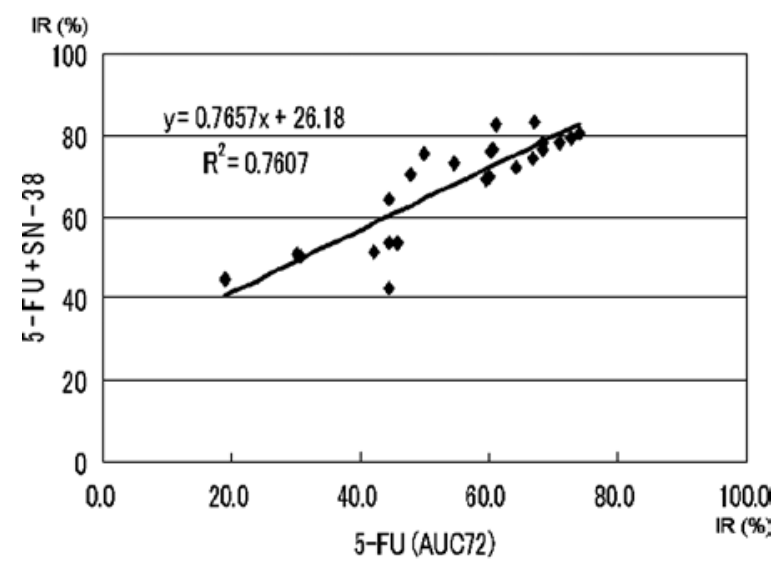

Figure 3. Effect of the addition of irinotecan (SN-38) to 5-fluorouracil (5-FU) on linear regression analysis. The growth inhibition rate (IR) of embedded cancer cells cultivated in culture media containing 5-FU at $3.0 \mu \mathrm{g}$ for $24 \mathrm{~h}$ vs. $5-\mathrm{FU}+\mathrm{SN}-38$ at $3.0+0.1 \mu \mathrm{g}$ for $24 \mathrm{~h}$.

Approximate expression and correlation coefficients on the AUC $(72 \mu \mathrm{g} * \mathrm{~h} / \mathrm{ml})$ of 5 -FU (5-FU vs. $5-\mathrm{FU}+l$-OHP and 5-FU vs. 5-FU + SN-38) were $\mathrm{y}=0.94 \mathrm{x}+8.53(\mathrm{p}<0.0004)$ and $\mathrm{y}=0.77 \mathrm{x}+26.18(\mathrm{p}<0.0004)$, respectively (Figs. 2 and 3$)$. 


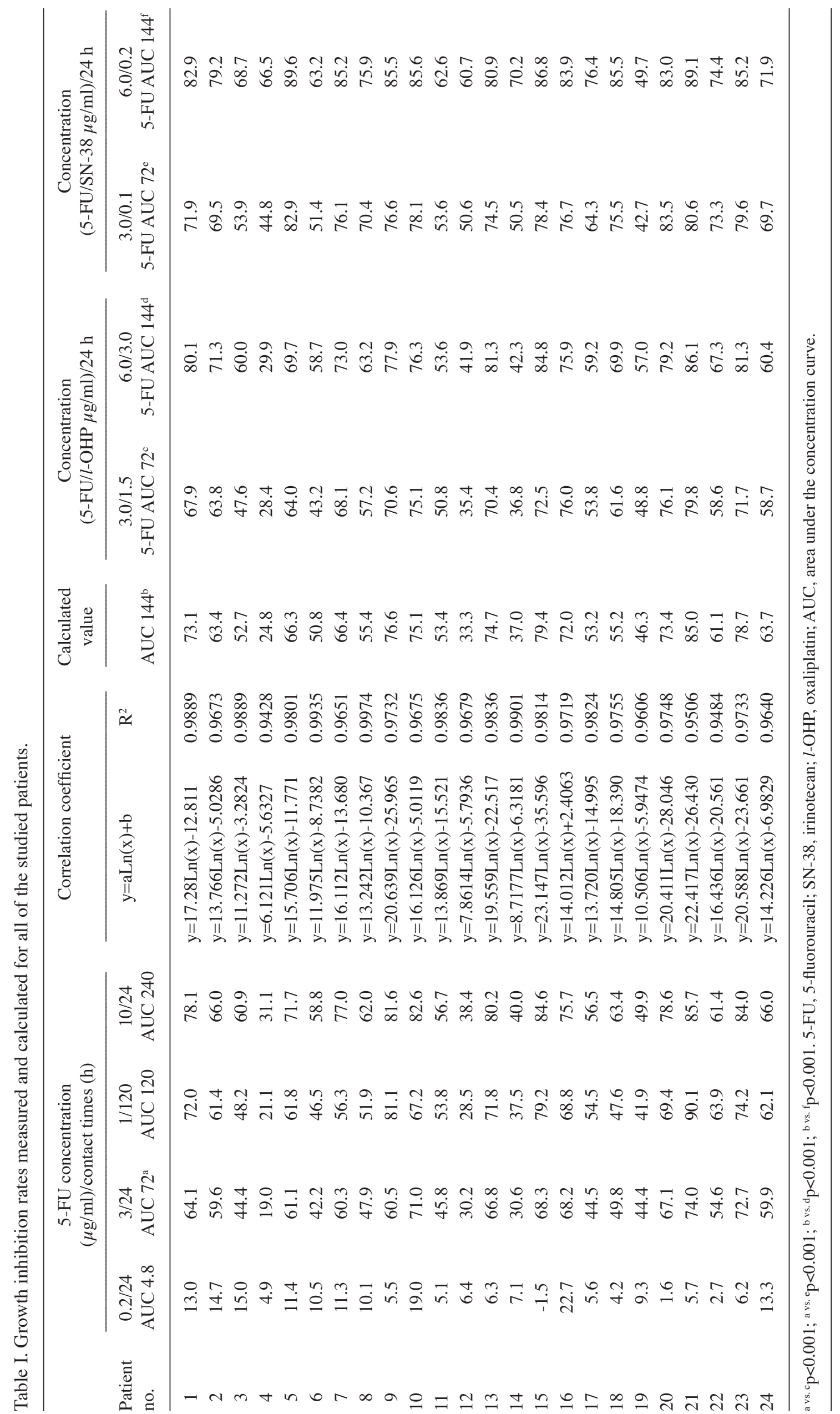




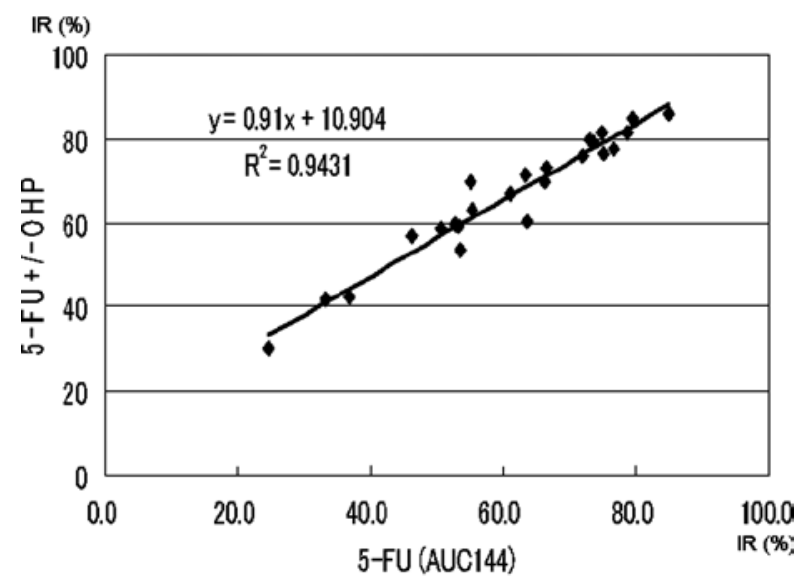

Figure 4. Effect of the addition of oxaliplatin ( $l$-OHP) to 5-fluorouracil (5-FU) on linear regression analysis. The calculated growth inhibition rate (IR) on the AUC $(144 \mu \mathrm{g} * \mathrm{~h} / \mathrm{ml})$ of 5 -FU vs. the growth IR of embedded cancer cells cultivated in culture media containing 5 -FU $+l$-OHP at $6.0+3.0 \mu \mathrm{g}$ for $24 \mathrm{~h}$.

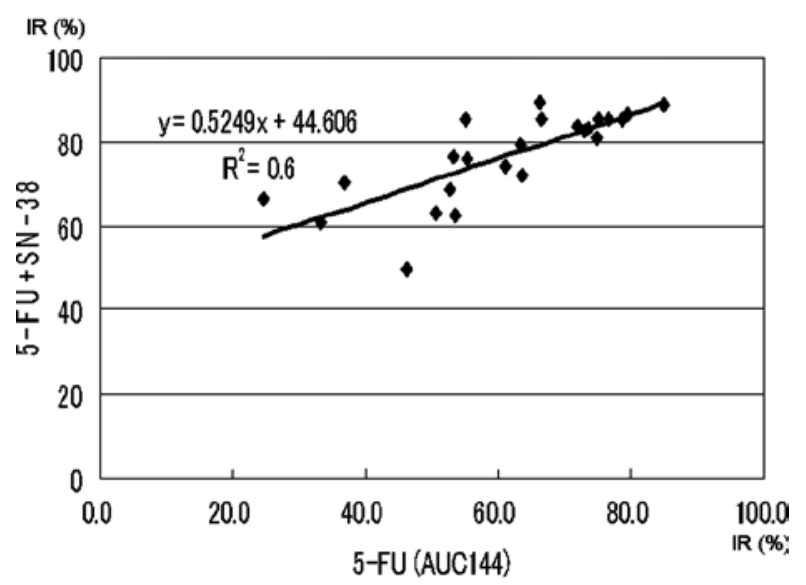

Figure 5. Effect of the addition of irinotecan (SN-38) to 5-fluorouracil (5-FU) on linear regression analysis. The calculated growth inhibition rate (IR) on the AUC $(144 \mu \mathrm{g} * \mathrm{~h} / \mathrm{ml})$ of 5-FU vs. the growth IR of embedded cancer cells cultivated in culture media containing $5-\mathrm{FU}+\mathrm{SN}-38$ at $6.0+0.2 \mu \mathrm{g}$ for $24 \mathrm{~h}$.
Approximate expression and correlation coefficients on the AUC $(144 \mu \mathrm{g} * \mathrm{~h} / \mathrm{ml})$ of $5-\mathrm{FU}(5-\mathrm{FU}$ vs. 5 -FU $+l$-OHP and 5-FU vs. 5 -FU $+\mathrm{SN}-38)$ were $\mathrm{y}=0.91 \mathrm{x}+10.90(\mathrm{p}<0.0004)$ and $\mathrm{y}=0.52 \mathrm{x}+44.61(\mathrm{p}<0.0004)$, respectively (Figs. 4 and 5$)$.

\section{Discussion}

In several randomized controlled trials, both FOLFOX and FOLFIRI have demonstrated improved patient survival as a first-line therapy in the treatment of advanced CRC (5-10). To obtain the same level of efficacy as that observed in these earlier trials, full administration of the targeted dosages of all three drugs (leucovorin and 5-FU $+l$-OHP or SN-38) is crucial (11). However, the pharmacokinetics of these drug combinations sometimes varies in CRC patients. Moreover, patients with a poorer performance status and, therefore, shorter life expectancy may have to be excluded from oxaliplatin- or irinotecan-based second-line chemotherapy. Therefore, since the best treatment for such patients may be either FOLFOX or FOLFIRI as first-line therapy, it is essential to be able to select which will be the most effective in each individual case.

In planning individualized chemotherapy, the drug sensitivity of the tumor cells is a key issue in assessing the anti-tumor effect of the anticancer drugs to be used. The CD-DST is a method of evaluating drug sensitivity using isolated, 3-dimensionally cultured tumor cells in a small collagen gel droplet (12). This method offers the following advantages: i) a high success rate in testing due to the micro3-dimensional culture; ii) the ability to work with a small quantity of specimen; iii) the ability to evaluate the anti-tumor effect of drugs in clinically equivalent doses; and iv) the ability to accurately evaluate anti-cancer effects using an image analysis device when fibroblast contamination is less than $67 \%$ (4). However, it is critical that the sample being examined is obtained from the soft part of the tumor tissue in order to prevent potential contamination by the fibroblast component. Determination of 5-FU exposure in this study was based on the report of Kobayashi (13). Determination of SN-38 and l-OHP exposure was based on the AUCs of 3 and 6 courses of FOLFOX or FOLFIRI. Using the CD-DST, we were able to calculate the individual growth IR value on the AUC $(144 \mu \mathrm{g} * \mathrm{~h} / \mathrm{ml})$ of 5 -FU from the individual AUC-IR curve in each patient.

In this study, approximate expression of 5-FU vs. 5-FU + $l$-OHP almost fit the regression line $\left(\mathrm{y}=\mathrm{x}+\mathrm{b}_{1}\right)$. This suggests that addition of $l$-OHP yields a constant effect, independent of the IR of 5-FU. This may be explained by the fact that the mechanisms of 5-FU and oxaliplatin function independently of each other. 5-FU is an S-phase-specific drug, and is only active during certain cell cycles. Three enzymes, in particular, are of great importance in the metabolism of 5-FU; orotate phosphoribosyl transferase (OPRT), thymidylate synthase (TS) and dihydropyrimidine dehydrogenase (DPD). OPRT is the most important phosphorylation enzyme of 5-FU; DPD is the degradation enzyme of 5-FU; and TS is the main enzyme of DNA synthesis (14). In general, poor efficacy for 5-FU is correlated with high expression of TS, whereas good efficacy for 5-FU is correlated with low expression of TS. Oxaliplatin is a cell-cycle non-specific antineoplastic agent (16). Therefore, addition of oxaliplatin yields a constant additive effect, independent of the IR of 5-FU.

On the other hand, irinotecan is an S-phase-specific drug like 5-FU and is only active during certain cell cycles (15). Approximate expression of 5-FU vs. 5-FU $+\mathrm{SN}-38$ fit the regression line $\left(y=a x+b_{2}, a<1, b_{2} \geq b_{1}\right)$. This suggests that addition of SN-38 yields a greater effect due to the lower growth IR of 5-FU. Therefore, there is a marked synergetic efficacy between 5-FU and irinotecan due to the lower growth IR of 5-FU. This synergy between irinotecan and 5-FU may be explained by prolonged inhibition of TS, down-regulation of TS mRNA expression and increased incorporation of 5-FU metabolites into DNA. There are several reports of the down regression of TS caused by irinotecan (17-22).

In conclusion, the results of this study suggest that FOLFIRI should be selected as first-line therapy in the treatment of poor responders to 5-FU. Moreover, it is suggested that, in planning individualized chemotherapy, it is vital to evaluate 
the efficacy of 5-FU when making the choice between FOLFOX or FOLFIRI as first-line treatment. In addition, it may be necessary to consider non-5-FU-based chemotherapy (i.e., irinotecan or oxaliplatin and molecularly targeted drugs) as the first-line treatment in cases where response to 5 -FU is extremely poor. Accurate evaluation of the efficacy of 5-FU is of the utmost importance in the establishment of individualized 5-FU-based chemotherapy.

\section{References}

1. Ochiai T, Nishimura K, Noguchi H, Kitajima M, Tsuruoka Y and Takahashi Y: Evaluation of 5-fluorouracil applicability by multipoint collagen gel droplet embedded drug sensitivity test. Oncol Rep 14: 201-205, 2005.

2. Ochiai T, Nishimura K, Noguchi H, et al: Evaluation of 5-fluorouracil applicability by the collagen gel droplet embedded drug sensitivity test with area under the curve analysis. Anticancer Drugs 18: 17-21, 2007.

3. Kobayashi H, Tanisaka K, Doi O, et al: An in vitro chemosensitivity test for soid human tumors using collagen gel droplet embedded cultures. Int J Oncol 11: 449-455, 1997.

4. Kobayashi H, Higashiyama $\mathrm{M}$, Minamigawa $\mathrm{K}$, et al: Examination of in vitro chemosensitivity test using collagen droplet culture method with colorimetric endpoint quantification. Jpn J Cancer Res 92: 203-210, 2001.

5. Tournigand C, André T, Achille E, et al: FOLFIRI followed by FOLFOX6 or the reverse sequence in advanced colorectal cancer: a randomized GERCOR study. J Clin Oncol 22: 229-237, 2004.

6. Falcone A, Ricci S, Brunetti I, et al: Gruppo Oncologico Nord Ovest. Phase III trial of infusional fluorouracil, leucovorin, oxaliplatin and irinotecan (FOLFOXIRI) compared with infusional fluorouracil, leucovorin and irinotecan (FOLFIRI) as first-line treatment for metastatic colorectal cancer: the Gruppo Oncologico Nord Ovest. J Clin Oncol 25: 1670-1676, 2007.

7. Saltz LB, Clarke S, Díaz-Rubio E, et al: Bevacizumab in combination with oxaliplatin-based chemotherapy as first-line therapy in metastatic colorectal cancer: a randomized phase III study. J Clin Oncol 26: 2013-2019, 2008.

8. Kabbinavar FF, Hambleton J, Mass RD, et al: Combined analysis of efficacy: the addition of bevacizumab to fluorouracil/leucovorin improves survival for patients with metastatic colorectal cancer. J Clin Oncol 23: 3706-3712, 2005.

9. André T, Louvet C, Maindrault-Goebel F, et al: CPT-11 (irinotecan) addition to bimonthly, high-dose leucovorin and bolus and continuous-infusion 5-fluorouracil (FOLFIRI) for pretreated metastatic colorectal cancer. GERCOR. Eur J Cancer 35: 1343-1347, 1999.
10. Maindrault-Goebel F, Louvet C, André T, et al: Oxaliplatin added to the simplified bimonthly leucovorin and 5-fluorouracil regimen as second-line therapy for metastatic colorectal cancer (FOLFOX6). GERCOR. Eur J Cancer 35: 1338-1342, 1999.

11. Grothey A, Sargent D, Goldberg RM, et al: Survival of patients with advanced colorectal cancer improves with the availability of fluorouracil-leucovorin, irinotecan and oxaliplatin in the course of treatment. J Clin Oncol 22: 1209-1214, 2004.

12. Matsuo A, Watanabe A, Takahashi T, et al: A simple method for classification of cell death by use of thin layer collagen gel for the detection of apoptosis and/or necrosis after cancer chemotherapy. Jpn J Cancer Res 92: 813-819, 2001.

13. Kobayashi H: Development of a new in vitro chemosensitivity test using collagen gel droplet culture and image analysis for clinical usefulness. Recent Results Cancer Res 161: 48-61, 2003.

14. Ochiai $\mathrm{T}$, Nishimura $\mathrm{K}$, Noguchi $\mathrm{H}$, et al: Prognotic impact of orotate phosphoribosyl transferase among 5-fluorouracil metabolic enzymes in resectable colorectal cancers treated by oral 5-fluorouracil-based adjuvant chemotherapy. Int J Cancer 118: 3084-3088, 2006.

15. Minderman H, Conroy JM, O'Loughlin KL, et al: In vitro and in vivo irinotecan-induced changes in expression profiles of cell cycle and apoptosis-associated genes in acute myeloid leukemia cells. Mol Cancer Ther 4: 885-900, 2005.

16. Lévi F, Metzger G, Massari C, et al: Oxaliplatin: pharmacokinetics and chronopharmacological aspects. Clin Pharmacokinet 38: 1-21, 2000.

17. Guichard S, Hennebelle I, Bugat R, et al: Cellular interactions of 5-fluorouracil and the camptothecin analogue CPT-11 (irinotecan) in a human colorectal carcinoma cell line. Biochem Pharmacol 55: 667-676, 1998.

18. Pavillard V, Formento P, Rostagno O, et al: Combination of irinotecan (CPT-11) and 5-fluorouracil with an analysis of cellular determinants of drug activity. Biochem Pharmacol 56: 1315-1322, 1998.

19. Banerjee D, Schnieders B, Fu JZ, Adhikari D, Zhao SC and Bertino JR: Role of E2F-1 in chemosensitivity. Cancer Res 58: 4292-4296, 1998.

20. Fukushima M, Uchida J, Sakamoto K, Ohshima H and Taguchi T: Molecular mechanism of down-regulation by CPT-11 of thymidylate synthase highly expressing in gastrointestinal cancer xenografts during combined treatment with fluoropyrimidines. Eur J Cancer 1: S62, 2003.

21. Ichikawa W, Takahashi T, Suto K, et al: Thymidylate synthase predictive power is overcome by irinotecan combination therapy with S-1 for gastric cancer. Br J Cancer 91: 1245-1250, 2004

22. Fukushima M: S-1 review from preclinical pharmacology. Gastric Cancer 12: 3-9, 2009. 